Final version published in:

D. Gorni, M. Castilla, J.D. Álvarez y A. Visioli (2015). "A comparison between Temperature Modeling Strategies in Smart Buildings". En: IEEE 20th Conference on Emerging Technologies \& Factory Automation (ETFA), Luxembourg.

DOI: $\underline{10.1109 / E T F A .2015 .7301596}$

http://ieeexplore.ieee.org/document/7301596/ 


\title{
A Comparison between Temperature Modeling Strategies in Smart Buildings
}

\author{
Domenico Gorni*, María del Mar Castilla ${ }^{\dagger}$, José Domingo Álvarez ${ }^{\dagger}$, Antonio Visioli ${ }^{\ddagger}$ \\ *Dipartimento di Ingegneria dell'Informazione \\ University of Brescia, Italy \\ Email: d.gorni001@unibs.it \\ $\dagger$ Department of Computer Science, University of Almería, \\ Agrifood Campus of International Excellence (ceiA3) and \\ CIESOL Joint Centre University of Almería-CIEMAT, \\ Almería, Spain \\ Email: \{mcastilla,jhervas\}@ual.es \\ $\ddagger$ Dipartimento di Ingegneria Meccanica e Industriale, \\ University of Brescia, Italy \\ Email: antonio.visioli@unibs.it
}

\begin{abstract}
This paper deals with modeling the temperature of a room in a smart building. Two different approaches are analyzed and compared. The first one is based on a black-box identification procedure, which yields a fairly simple dynamical system that is suitable for real-time control. The second approach is based on first principles, it requires a complex and time consuming calibration procedure but it is capable of describing accurately the physical behavior of the system. The trade-off between the accuracy of the model and its computational complexity is evaluated by using experimental data collected from a smart pilot building located in Almería, Spain.
\end{abstract}

\section{INTRODUCTION}

Nowadays, people spend most of their time developing their quotidian activities inside buildings. For this reason, it is necessary to provide comfortable environments while, at the same time, trying to satisfy the energy requirements which have been established in the different parts of the world. Indeed, the energy consumption in buildings represents approximately $40 \%$ of the total world energy consumption [1] and therefore this issue is very relevant to achieve a sustainable growth. In order to reach the required goals, one of the most used options consists of developing appropriate control strategies that are able both to optimize the energy consumption and to maintain the users' comfort (see, for example, [2], [3]). In this context, temperature control plays a major role (although also air quality is very important to determine the comfort) and it is therefore necessary to develop appropriate models since they can provide useful information about the behavior of different control systems avoiding the associated costs and risks derived from testing them in a real plant. In other words, an accurate model can be used either for simulation and evaluation purposes or for the design of (model-based) control systems [4]. Within the process control area, it is very usual to develop nonlinear first principles models of the system. However, mainly because of the excessive computational complexity, it can be more sensible to obtain linear and nonlinear models by means of classical identification techniques.

In general, the majority of the available models which can be found in literature are related to the evaluation of the energy performance in buildings (see, for example, [5], [6]), and to the development of adequate control strategies at building level (see, for example, [7]). Furthermore, in literature there are works which use different techniques to obtain black-box models such as Artificial Neural Networks (ANNs) [8]. As a result, it is possible to find different kinds of models which are developed under several perspectives and with diverse final objectives.

In this paper we perform a comparison between the computational complexity and accuracy provided by two indoor air temperature models. In particular, we consider a recently devised black-box model whose parameters are estimated by means of a standard identification procedure and we compare it with a (complex) nonlinear model based on first principles.

The comparison is performed by analyzing the main characteristics of the two methods and by evaluating the results obtained by using experimental data collected in a room inside the CIESOL building (http://www.ciesol.es/en), which is a smart building located in Almería, Spain. The aim of the comparison is to provide a trade-off between the accuracy and computational complexity of two different strategies, so that the user can evaluate the most convenient one to be used depending on the required task.

\section{INDOOR AIR TEMPERATURE MODELS}

In general, the development of appropriate models that are capable to precisely represent the dynamic behavior of a system requires both effort and time. A room is a complex system influenced by different kinds of elements and by the surroundings environmental conditions. Furthermore, these elements can be defined as a function of their thermal and optical properties. In this section, two different approaches to 
obtain a room-level indoor air temperature model are shown, a black-box model (see [9]) and a nonlinear first principles model.

\section{A. Black-box Model}

The proposed approach consists in considering a single room and in determining an (black-box) Auto-Regressive Moving Average with eXogenous inputs (ARMAX) model based on the first-principles thermodynamics equations of the system. Then, the model parameters are estimated by initially employing a standard procedure and then by using a selfcalibrating method when the performance deteriorates.

The thermodynamic fundamental equation that describes the variation of the temperature of a single mass is:

$$
m_{a} C_{p_{a}} \frac{d T_{a i n}}{d t}=Q
$$

where $Q[W]$ is the total amount of heat exchanged, $C_{p_{a}}$ $\left[\mathrm{Jkg}^{-1} \mathrm{~K}^{-1}\right]$ is the specific heat, $m_{a}[\mathrm{~kg}]$ is the mass, $T_{\text {ain }}$ $[K]$ is the temperature and $t[s]$ is the time. By applying this equation to a room it is possible to describe the variation of its temperature by taking into account simplifying assumptions, such as: (i) the air inside the room is supposed to be at the same temperature; (ii) the room is considered a closed system with no mass exchange with the external environment. However, a single room is usually bordering with more different areas, for example with the ground through the floor, or with the external environment or with other rooms. Thus, it is also important to take into account all the different heat flows to estimate the temperature evolution of a room. Moreover, heat sources like Heating, Ventilation, and Air Conditioning (HVAC) systems that can heat or cool the room are also usually present.

Considering the six main dimensions of a parallelepipedal room, the heat balance between the room and the surrounding spaces can be described as:

$$
Q=\sum_{i=1}^{6} q_{i}=\sum_{i=1}^{6} \frac{1}{R_{i}}\left(T_{a i n}-T_{s i}\right) \quad i=1 \ldots 6
$$

where $R_{i}\left[W K^{-1}\right]$ is a coefficient that represents the combination between convection and conduction resistances of the $i$ th surface, $T_{s i}$ is the $i$ th surrounding space temperature (that is, $T_{s i}$ can be $T_{N}, T_{S}, T_{W}, T_{E}, T_{f}$ or $T_{c}$ ) and $T_{a i n}$ is the indoor room temperature (all in $[K]$ ). The differential equation that describes the temperature evolution is:

$$
m_{a} C_{p_{a}} \dot{T}_{a i n}=\sum_{i=1}^{6} q_{i}+\sum_{i=7}^{m} q_{i}=\sum_{i=1}^{6} \frac{1}{R_{i}}\left(T_{a i n}-T_{s i}\right)+\sum_{i=7}^{m} q_{i}
$$

where $q_{1} \ldots q_{6}$ are the heat flows gained and lost to the surrounding walls and $q_{7} \ldots q_{m}$ are the exogenous heat sources like HVAC systems, the direct radiation from the sun, electrical devices, etc.

The aim is to write a thermal model of the room that requires simple sensors, that is, based on:

- the measure of temperature of the adjacent rooms;

- the measured heat flow of the exogenous inputs.
Now the model will be rewritten to be suitable for control purposes. Therefore, the first step is to apply the Laplace transform to the equation (3). Then, a Tustin bilinear transform is applied to the continuous transfer function in order to obtain an ARMAX black-box model [9], that is:

$\hat{T}_{a i n}(k)=\sum_{j=1}^{8}\left\{\mathbf{g}_{\mathbf{j}}(\mathbf{T}(k-j+1)+\boldsymbol{\varepsilon}(k-j+1))+\mathbf{p}_{\mathbf{j}} \mathbf{q}(k-j+1)\right\}$

where $\hat{T}_{a i n}$ is the estimated value of the indoor air temperature, $\varepsilon$ denotes the measurement noises and $\mathbf{g}_{j}$ and $\mathbf{p}_{j}, j=1, \ldots, 8$ are vectors of suitable coefficients. The unknown parameters estimation can be done with the application of the Matlab function called armax; this function minimizes a robustified quadratic prediction error criterion by using an iterative GaussNewton algorithm [10]. This method is applied on (4) to determine all the $\mathbf{g}_{\mathbf{j}}$ and $\mathbf{p}_{\mathbf{j}}$ unknown parameters. Even though this equation is a direct consequence of the thermodynamical equations, during all the passages the parameters have lost their physical meaning, in fact such ARMAX models are defined black-box models.

More specifically, the estimation methodology consists in estimating an initial set of parameters with the data collected in the first day of operations. Then, a recalibration procedure is applied to the model if the model accuracy is no more satisfactory. Details of this procedure as well as practical issues are given in [9].

It is worth stressing at this point that the computational complexity of the model is quite low. In order to keep it as simple as possible, the only exogenous inputs that have been adopted are the temperature of the fancoil air (which can be substituted in general with the output of the feedback temperature controller), the solar radiation, and the temperatures of the air of the adjacent rooms. In the particular case of the CIESOL building (see Section III) these latters have been substituted with the temperatures of the walls of the considered room, as they were the only available sensors. However, it has to be noted that the employed method is virtually the same (see Section III).

\section{B. Nonlinear Model based on First Principles of Thermody- namics}

In order to obtain a nonlinear first principles model, the existing relations among the elements which compose the room and between them and the environment have been modeled by means of heat and mass transfer laws. More concretely, a model has been developed using the so-called "Heat Balance Method" described by [11]. Furthermore, it has been subjected to the following assumptions: (i) the room has been considered to be composed of seven elements: indoor air, walls, windows, shading system, HVAC system, people and electrical appliances; (ii) the air inside the room has been considered as a perfect mix, that is, it is supposed that the air temperature inside the room is uniform in the whole room [12]; (iii) it has been supposed that the surfaces of the room have a uniform temperature, similar wave irradiance 
and a one-dimensional heat conduction process; The physical characteristics of the different elements of the room, with the exception of the indoor air, has been assumed to be constant. As a consequence, the indoor air temperature has been modeled by means of a differential equation based on mass and heat transfer principles as it is shown in [13].

This system has a set of nine unknown parameters whose values have been determined by means of a specific calibration methodology. The values obtained for these parameters after the calibration process are the same along the whole year. More specifically, the calibration methodology is a cascade process consisting of, in an initial approach, a brute force sequential search to estimate a first approximation for the unknown parameters, and then, the use of evolutionary algorithms to obtain the final value. In particular, in order to obtain the initial approximation of the unknown parameters, different tests for each one of the involved processes have been performed all around the year. In this context, it has been necessary to separate the room into elemental systems and different tests have been performed with the aim to separately determine the influence of each element in the indoor temperature. Each one of these tests has been performed in several days of a certain season of the year. Afterwards, and based on the reference values of the guidelines provided by [11], the first approximation of the unknown parameters has been calculated by using a brute force sequential search algorithm. Subsequently, a search technique based on genetic algorithms has been used to determine the final value of the unknown parameters. It appears that the overall modeling methodology considers almost all the physical issues involved in the process but it is quite complex to implement in practical cases and the obtained model can be too computational demanding to be employed in a real-time controller.

\section{THE CIESOL BUILDING}

The CIESOL building is a reference research center on solar energy, situated in the Campus of the University of Almería, in the southeast of Spain (see [13] for details). In order to calibrate and validate the models proposed in this paper, a typical room of this building has been selected. This room is situated on the top floor of the CIESOL building between two rooms with analogous characteristics. More specifically, it has north orientation, a total volume of $76.8 \mathrm{~m}^{3}$ and a window of $4.49 \mathrm{~m}^{2}$ located in the north wall. Besides, it is characterized by the presence of a huge diversity of sensors (air temperature, air velocity, plane radiant temperature, humidity, etc.) and a wide set of actuators, such as a fancoil, a shading system and a window opening/closing system, that are used in general to control users' comfort. In particular, there is a plane radiant temperature sensor for each wall, therefore for the black-box model temperatures of the walls of the room have been employed instead of the temperatures of the adjacent rooms (as well as for the first principles model, where these have been already explicitly considered). In fact, the technique described in Section II-A can be straightforwardly applied in this case by substituting the term $1 / R_{i}$ in (2) and (3) with $h_{i} A_{i}$ where $h_{i}\left[W m^{-2} K^{-1}\right]$ and $A_{i}\left[m^{2}\right]$ are, respectively, the convective coefficient and the area of the $i$ th wall surface.

\section{RESULTS AND DISCUSSION}

The obtained models have been validated by using the following three new datasets collected in different periods of the year, in order to verify the effectiveness of the modeling strategies under several circumstances and, in particular, with different outdoor conditions (cold, warm and hot weather, taking into account the geographical position of the building). Actually, while the same first principles model has been employed for all the datasets, the ARMAX model has been recalibrated for the third dataset (by using the data collected in the three days before the period of the validation set) because the results were not satisfactory. Indeed, this confirms the need for the black-box model to be recalibrated when the season changes (note that the original model has been calibrated during the winter while the third validation dataset has been collected during the hot season).

- Validation dataset 1 (cold weather). This test was performed between 12th and 22nd of December in 2013 under different environmental conditions. It contains some periods when the room was empty and others with the presence of its usual occupants. Besides, along this test some controlled experiments have been performed using the actuators available inside the room, that is, the HVAC system and the window. The results provided by this validation test are shown in Fig. 1. The real variable (measured inside the typical room of the CIESOL building) is plotted in blue, the results provided by the first principles model in red, and finally, the results obtained from the ARMAX model in green.

- Validation dataset 2 (warm weather). This test was performed between 4th and 10th of March in 2013 under similar conditions: windows and door were closed, with several disturbances due to the inputs and outputs of people and the blind was open. Hence, this dataset also contains some periods when the room was empty and others with the presence of its usual occupants. In addition, one of the main characteristics of this dataset is that it is an intermediate period, and thus, there was no need of using any actuator. The results provided by this validation dataset can be observed in Fig. 2 where the same colors of the previous case have been employed.

- Validation dataset 3 (warm/hot weather). This test was performed between 10th and 12th of May in 2013 under varying conditions. As in the previous validation datasets, it contains some periods where the room was empty and others with the presence of its usual occupants. Moreover, along this test some controlled experiments using the actuators available inside the room have been performed. More specifically, on the 10th of May the HVAC system was used, and the window was opened several times on 11th and 12th of May. The results obtained from validating the proposed models with this validation set are shown in Fig. 3. 


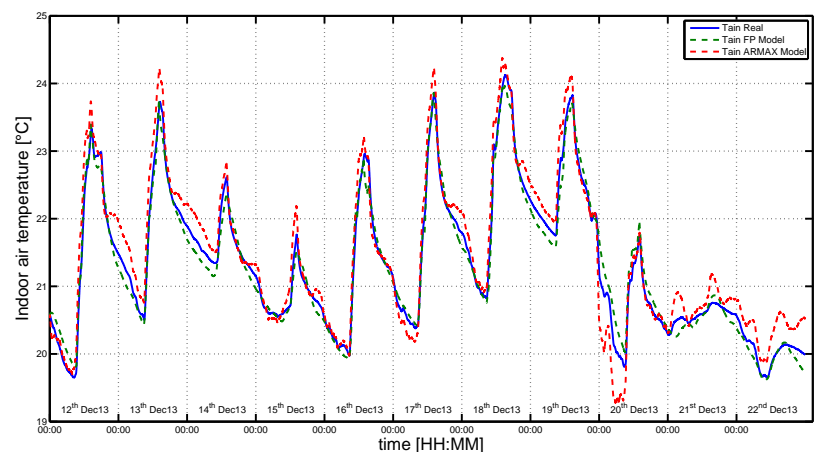

Fig. 1. Results of validation dataset 1 .

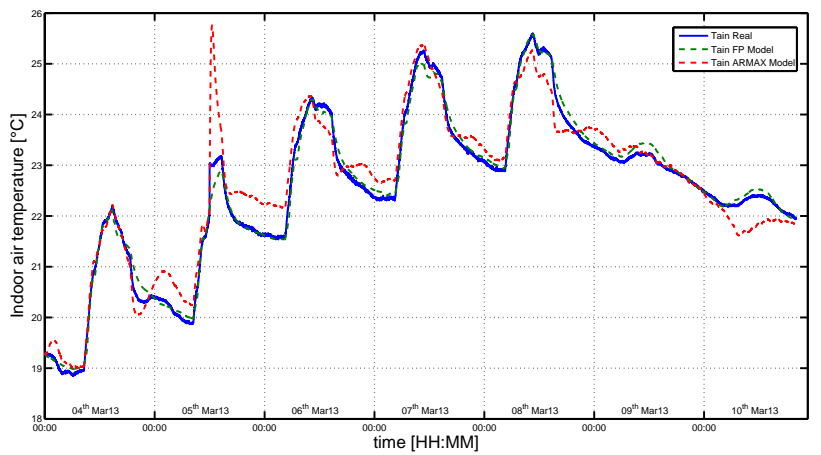

Fig. 2. Results of validation dataset 2 .

Even if both models can describe the real system accurately, the first principles model has a better performance in two of the three tests, namely Test 1 and Test 2 which are the ones with the greatest range and the largest number of samples. In Test 3 the black-box ARMAX model is simulating the evolution of the room temperature with a smaller error because of the retuning performed in the previous days. Summarizing, the general worst performance in simulating the evolution of the system provided by ARMAX model is balanced by the difference between the computational times; the first principles model is always at least 50 times slower than the other one. Indeed, the computational time for the execution of the proposed models with each one of the three validation tests (performed with a computer with an Intel Core i3 processor

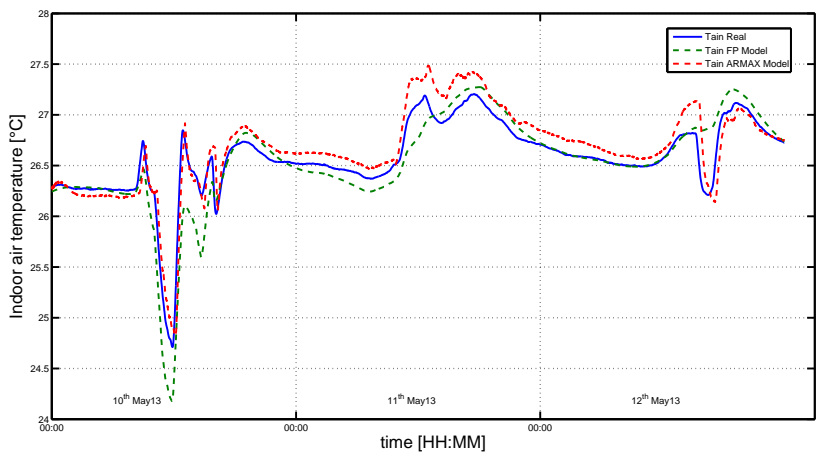

Fig. 3. Results of validation dataset 3 . at $3.30 \mathrm{GHz}, 4 \mathrm{~GB}$ of memory and Windows 7 as operating system) are, respectively, $1.2 s, 0.76 s, 0.40 s$ for the ARMAX model and $104.14 s, 64.49 s, 28.59 s$ for the first principle models.

\section{CONCLUSIONS}

This paper has compared the performance of two completely different approaches to solve the same temperature modeling problem. The first principles model has the advantage to have a clear physical meaning and therefore, after having been properly calibrated, it can describe effectively the behavior of the real system in all the different periods of the year. On the contrary, the black-box modeling approach is a faster way to describe the same system and, thanks also to an online parameters self-calibrating technique, can reach a good approximation level. Both of these models can be used in high level optimal controllers, the first principles model is a better representation of the real system while the ARMAX model can be useful when a real-time implementation is needed.

\section{ACKNOWLEDGMENT}

Authors wish to thank the financial support provided by the Transnational Access to Research Infrastructures within the European project SFERA II (Grant Agreement n. 312.643) under the 7th Framework Program.

\section{REFERENCES}

[1] L. Pérez-Lombard, J. Ortiz, and C. Pout, "A review on building energy consumption information," Energy and Buildings, vol. 40, pp. 394 398, 2008.

[2] A. Kelman, Y. Ma, and F. Borrelli, "Analysis of local optima in predictive control for energy efficient buildings," in Proceedings of 50th IEEE Conference on Decision and Control and European Control Conference, Orlando,FL,USA, 2011, pp. 5125-5130.

[3] P. M. Ferreira, A. E. Ruano, S. Silva, and E. Z. E. Conceico, "Neural networks based predictive control for thermal comfort and energy savings in public buildings," Energy and Buildings, vol. 55, pp. 238-251, 2014.

[4] P. Radecki and B. Hencey, "Online thermal estimation, control, and self-excitation of buildings," in Proceedings of 52th IEEE Conference on Decision and Control, Florence (I), December 2013, pp. 4802-4807.

[5] T. Olofsson and T. M. I. Mahlia, "Modeling and simulation of the energy use in an occupied residential building in cold climate," Applied Energy, vol. 91, pp. 432-438, 2012.

[6] D. Saelens, W. Parys, and R. Baetens, "Energy and comfort performance of thermally activated building systems including occupant behaviour," Building and Environment, vol. 46, pp. 835 - 848, 2011.

[7] I. Hazyuk, C. Ghiausa, and D. Penhouet, "Optimal temperature control of intermittently heated buildings using model predictive control: Part i. building modeling," Building and Environment, vol. 51, pp. 379 - 387, 2012.

[8] M. Castilla, J. D. Álvarez, M. G. Ortega, and M. R. Arahal, "Neural network and polynomial approximated thermal comfort models for HVAC systems." Building and Environment, vol. 59, pp. 107-115, 2013.

[9] D. Gorni and A.Visioli, "Practical issues in modelling the temperature for the control of smart buildings," Journal of Physics: Conference Series, vol. 570, 2014.

[10] L. Ljung, System Identification Toolbox: User's Guide. Natick, MA: The Mathworks, 2014.

[11] ASHRAE, ASHRAE Handbook - Fundamentals. Refrigerating American Society of Heating and Air-Conditioning Engineers, 2009.

[12] D. E. Fisher and C. O. Pedersen, "Convective heat transfer in building energy and thermal load calculations," ASHRAE Transactions, vol. 103(2), pp. 137-148, 1997.

[13] M. Castilla, J. D. Álvarez, F. Rodríguez, and M. Berenguel, Comfort control in buildings. Springer. Advances in Industrial Control Series, 2014. 